\title{
Real-time optical path difference compensation at the Plateau de Calern I2T interferometer
}

\author{
B. Sorrente ${ }^{1}$, F. Cassaing ${ }^{1}$, G. Rousset ${ }^{1}$, S. Robbe-Dubois ${ }^{2,3}$, and Y. Rabbia ${ }^{2}$ \\ 1 Office National d'Études et de Recherches Aérospatiales (ONERA), DOTA, BP 72, 92322 Châtillon Cedex, \\ France \\ e-mail: sorrente@onera.fr \\ 2 Observatoire de la Côte d'Azur, Fresnel Department, UMR 6528, avenue Copernic, 06130 Grasse, France \\ 3 now at Université de Nice - Sophia Antipolis, Laboratoire d'Astrophysique, UMR 6525, Parc Valrose, \\ 06108 Nice Cedex 2, France \\ e-mail: Sylvie.robbe-dubois@unice.fr
}

Received 6 January 1999 / Accepted 14 August 2000

\begin{abstract}
The fringe tracker system of the ASSI (Active Stabilization in Stellar Interferometry) beam combining table at the I2T interferometer is described and its performance evaluated. A new real-time algorithm for the optical path difference (OPD) measurement is derived and validated. It is based on a sinusoidal phase modulation whose amplitude is optimized. It also allows automatic fringe detection at the beginning of an observation when scanning the OPD. The fringe tracker servo-loop bandwidth is adjusted by a numerical gain and ranges between 20 and $50 \mathrm{~Hz}$ in the reported experiments. On stars, fringe-locked sequences are limited to $20 \mathrm{~s}$ due to fringe jumps. However, the fringe tracker is able to recover the coherence area after a few seconds. Such a fringe tracker operation can last more than one hour. A fringe tracking accuracy of $85 \mathrm{~nm}$ is achieved for visibility ranging between 7 and $24 \%$, a turbulence coherence time of approximately $9 \mathrm{~ms}$ at $0.85 \mu \mathrm{m}$, a Fried parameter of around $14 \mathrm{~cm}$ at $0.5 \mu \mathrm{m}$ and an average light level of 100000 photoevents/s, (typically visual magnitude 2 in the conditions of the experiment). Visibility losses are evaluated and are found to be mainly due to turbulent wavefront fluctuations on the two telescopes and to the static aberrations of the optical train. The measurements of OPD and angle of arrival are reduced to derive turbulence parameters: the coherence time, the average wind speed, the Fried parameter and the outer scale. Our estimations for the outer scale range between 20 and $120 \mathrm{~m}$, with an average value of the order of $40 \mathrm{~m}$. Both OPD and angle of arrival data, obtained on $15 \mathrm{~m}$ baseline and a $26 \mathrm{~cm}$ telescope diameter respectively, are fully compatible with the same modified Kolmogorov spectrum of the turbulence, taking into account a finite outer scale.
\end{abstract}

Key words. atmospheric effects - instrumentation: interferometers - methods: data analysis methods: observational - techniques: interferometric

\section{Introduction}

According to the Zernike-van Cittert theorem, the fringe visibility in a stellar interferometer of baseline $\mathbf{B}$ is directly related to the object spectrum value at the spatial frequency $\mathbf{B} / \lambda$ where $\lambda$ is the observation wavelength. Since the baseline can reach several tens of meters, long baseline interferometry has the ability to achieve very high resolution (Labeyrie 1975). However, observations made from the ground are severely limited by atmospheric turbulence (Roddier 1981). Such turbulence induces wavefront disturbances over the telescopes, reducing the fringe visibility. These distorsions are responsible for speckled images, image motion and optical path difference (OPD) fluctuations. Turbulence effects can be fought by speckle

Send offprint requests to: B. Sorrente techniques, based on the recording of a long set of short exposure data, or by real-time correction for better signal to noise ratio (SNR) (Roddier \& Léna 1984). Two modes can be distinguished for OPD correction. In the coherencing mode, the requirement is to achieve OPD residuals smaller than the coherence length, ensuring interference only in the short exposure data. In the cophasing mode, the requirement is to achieve OPD residuals much smaller than the wavelength, allowing long exposures. In case of large OPD residuals, cophasing suffers from fringe jumps and requires some kind of coherencing to track the central fringe. Only a few fringe tracker systems have been implemented for stellar observations. The SUSI (Davis et al. 1995) or GI2T (Koechlin et al. 1996) interferometers allow only coherencing. Cophasing (with simultaneous coherencing) has only been used by the Mark III 
(Shao et al. 1988), NPOI (Armstrong et al. 1998) or PTI (Colavita et al. 1999) interferometers.

The "Active Stabilization in Stellar Interferometry" (ASSI) table, developed by the Office National d'Études et de Recherches Aérospatiales (ONERA) aims to compensate in real-time for turbulence disturbances in the "Interféromètre à 2 Télescopes" (I2T) of the Observatoire de la Côte d'Azur (OCA), France (Robbe et al. 1994). OPD and angle of arrival fluctuations respectively are corrected by a fringe tracker and two star trackers, one for each telescope. The first fringes were observed and stabilized in June 1994 with an $11 \mathrm{~m}$ baseline.

The goals of our work were to implement the technologies relevant to optical aperture synthesis and to demonstrate the performance of fringe tracking as a function of observing conditions, i.e. the seeing and the visual magnitude $\left(m_{\mathrm{V}}\right)$ of the observed star. The performance evaluations are coupled to measurements of the spatial and temporal characteristics of the turbulence. Atmospheric measurements previously were made by other stellar interferometers (Mariotti \& Di Benedetto 1984; Bester et al. 1992; Buscher et al. 1995; Davis et al. 1995).

This paper discusses the results obtained with the fringe tracker. For the star trackers, see Robbe et al. 1997. In Sect. 2, the I2T-ASSI interferometer and the fringe tracker are described. In Sect. 3, two new algorithms for fringe detection and tracking are presented and their noise performance given. An analysis of visibility losses is performed in Sect. 4. The experimental visibilities are compared with the expected values. Section 5 deals with the fringe tracking results in the laboratory and the sky. In Sect. 6, we report the estimations of the atmospheric coherence time and the outer scale deduced from temporal power spectra and variances of OPD and angle of arrival fluctuations.

\section{Description of the instrument}

\subsection{The I2T-ASSI interferometer}

I2T is a stellar interferometer including two movable $26 \mathrm{~cm}$ diameter telescopes mounted on rail tracks on a northsouth baseline (Koechlin \& Rabbia 1985). Accessible baselines span from 10 to $140 \mathrm{~m}$. Light is sent through the air towards a central laboratory where the two beams are combined by ASSI for visibility measurements in a scientific instrument. The path length variation due to the Earth's rotation is compensated by a cat's eye delay line inserted in the south arm. A static delay line in the north arm ensures symmetry.

Early I2T operations showed the limitations of visual fringe search and calibration of turbulence-degraded visibility (Koechlin 1985). It was decided in 1988 to equip I2T with the ASSI table dedicated to automatic fringe detection and fringe stabilization (Damé et al. 1988; Sorrente et al. 1991). Its design was partially upgraded before the instrument was set up at I2T in 1993 (Sorrente et al. 1994).

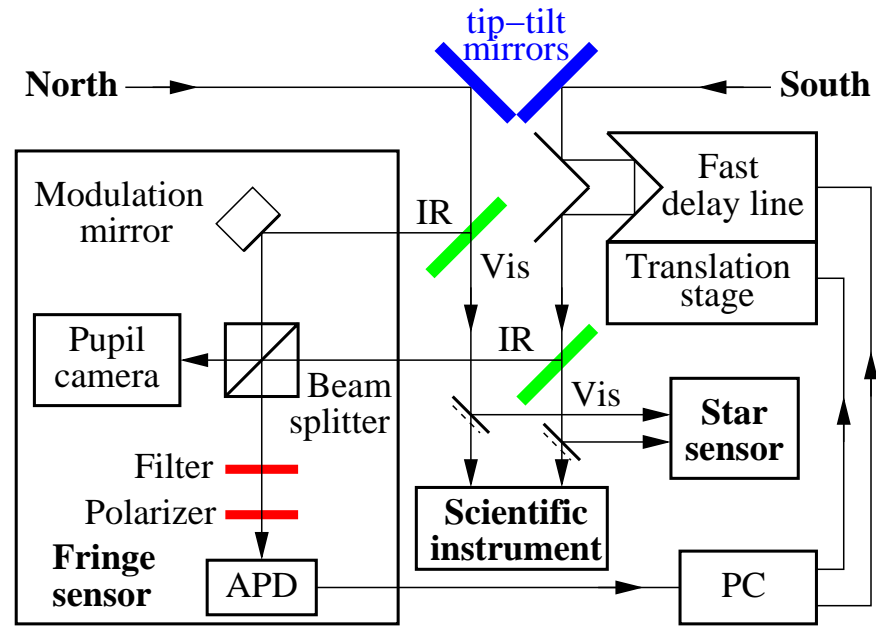

Fig. 1. Block diagram of the fringe tracker system of ASSI

The ASSI table has already been presented by Robbe et al. 1997. It mainly involves two servo-tracking systems dedicated to the angle of arrival correction of the two telescopes, and one dedicated to OPD correction (Fig. 1). The star trackers share the visible light with a scientific dedicated instrument in the $0.4-0.78 \mu \mathrm{m}$ spectral range. A photon-counting quad cell detector (star sensor), alternatively fed by each arm of the interferometer, generates the error signals used to command the tip-tilt mirrors. The system and its performance have been fully described in a previous paper (Robbe et al. 1997): an accuracy of \pm 0.24 arcsec has been achieved for a light level of 50000 photoevents/s (typically $m_{\mathrm{V}}=2$ ) and $r_{0} \geq$ $10 \mathrm{~cm}$ at $0.5 \mu \mathrm{m}$, where $r_{0}$ is the Fried parameter. The three servo-systems are driven by a $486 / 25 \mathrm{MHz}$ Personal Computer (PC), allowing data acquisition from the star and fringe trackers and providing an user interface.

The scientific instrument is based on dispersed Young-type fringes, providing a two dimensional OPDwavelength interferogram. This fringe pattern is recorded on a photon-counting camera with an exposure time of $20 \mathrm{~ms}$ and then 2-D Fourier transformed to extract the visibility.

\subsection{The fringe tracker}

Once the two beams are tilt-stabilized, their red component $(0.78-1 \mu \mathrm{m})$ is sent towards the fringe sensor. The purpose of the fringe tracker is first to localize the coherence area and then to compensate for the OPD between the beams, induced by both atmospheric turbulence and metrology errors.

To measure phase and visibility, a method widely used is to generate a known phase modulation in the interferometer and to synchronously demodulate the intensity variations. In stellar interferometry, the measurement time must be short enough to freeze the fringe motion induced by atmospheric turbulence (a few milliseconds in the visible). The magnitude of the observed objects requires high 
efficiency detectors and wide band observation. These constraints lead to the choice of a coaxial beam combination with sinusoidal temporal phase modulation and a photoncounting avalanche photodiode (APD), manufactured by EG\&G, as a single-pixel detector.

In the fringe sensor (Fig. 1), the two afocal beams are superimposed by a beam splitter cube in a flat-tint mode, in a pupil plane, as in the Mark III interferometer (Shao et al. 1988). One of the two complementary outputs is used for interference state measurement, with the APD. A filter allows selection of the spectral bandpass. A camera for diagnostic purpose, such as pupil lateral positioning, is set up at the other output. In the north arm of the fringe sensor, a mirror mounted on a piezoelectric (PZT) actuator induces a $280 \mathrm{~Hz}$ OPD modulation. This PZT is operated in open-loop conditions, but its transfer function is measured with an internal calibration source before observation. Real-time visibility and phase measurements are obtained by demodulation of the APD signal (described in Sect. 3) during the observation. A single interrupt-driven routine in the $\mathrm{PC}$ is in charge of PZT driving modulation, APD counter reading, demodulation and control of the OPD correction device, ensuring simple and perfect synchronization.

A first-order integral controller with adjustable gain is used to derive the OPD command from the phase measurements (Appendix A). The numerical loop-gain is adjusted by the observer according to the observing conditions (light level and turbulence) in order to minimize the OPD residual variance. However, an automatic procedure would have been much more efficient to obtain the best optimization. The correction device is a two-stage delay line, including a roof mirror mounted on a $\pm 8 \mu \mathrm{m}$ mechanical stroke PZT actuator (fast delay line) and a $\pm 10 \mathrm{~mm}$ micropositioning translation stage. The correction signal is sent to the PZT actuator. When the PZT elongation exceeds a threshold value, the translation stage is used for desaturation.

The visibility measured by the fringe sensor (Sect. 3) is used for fringe detection by comparison with a threshold value selected by the observer and based on the visibility level measured at large OPD (incoherent measurement). The fringe detection process works continuously as the OPD is linearly scanned by the translation stage. The integration time is set according to the coherence length $L_{\mathrm{c}}$ of the fringe pattern and the scan speed. Typical values are $L_{\mathrm{c}}=9 \mu \mathrm{m}$ and a few $\mu \mathrm{m} \mathrm{s}^{-1}$, respectively, allowing an integration time of a few seconds. If the visibility estimate increases above the alert threshold during the OPD scan, then the scan is stopped. A new visibility estimate, with a longer integration time, is performed. Comparison with another threshold, set for these new conditions, allows the system to decide whether fringes are actually there or not. In case of fringe detection, the servo tracking is automatically switched on, otherwise the scan resumes.

\section{Visibility and phase measurement technique}

The spectral bandwidth of the source and fringe sensor produces the following interferogram:

$I(\Psi)=I_{0}[1+V(\Phi+\Psi) \cos (\Phi+\Psi)]$

where $I$ is the measured intensity, $I_{0}$ the mean intensity, $V(\Phi)$ the visibility. $\Phi$ is the phase related to the position of the fringes proportional to the OPD between the two telescopes, and $\Psi$ the phase modulation. For monochromatic light, $V$ is independent of $\Phi$ and the interferogram can be detected with small amplitude $(\simeq \lambda)$ modulation. For polychromatic light, the fringe pattern has an envelope: $V$ is high only within the coherence area, and null outside.

In the cophasing mode, assuming that the modulation amplitude is much smaller than the coherence length, the polychromatic interferogram can be approximated by a monochromatic interferogram of visibility $V(\Phi+\Psi) \simeq$ $V(\Phi)$. Equation (1) then becomes:

$I(\Psi)=I_{0}+I_{0} V(\Phi) \cos \Phi \cos \Psi-I_{0} V(\Phi) \sin \Phi \sin \Psi$.

The modulated intensity is thus the sum of the incoherent intensity offset and two interferometric signals, proportional to the known waveforms $\cos \Psi$ and $\sin \Psi$. By linear demodulation, it is possible to estimate $I_{0}$ and the two fringe quadratures $I_{0} V(\Phi) \cos \Phi$ and $I_{0} V(\Phi) \sin \Phi$, from which fringe parameter estimation is straightforward, the phase being measured modulo $2 \pi$. To allow a high modulation frequency, a continuous OPD modulation is most often used, while pulses delivered by the photon-counting detector are integrated in $K$ temporal buckets.

Taking advantage of the orthogonality of the trigonometric functions, a triangle OPD modulation of amplitude $\lambda$ is usually chosen, followed by a Digital Fourier Transform of the $K$ samples $\left(\mathrm{DFT}_{K}\right)$. This is the case of the so-called ABCD algorithm with $K=4$ intensity buckets per modulation period (Shao et al. 1988).

\subsection{Noise propagation for demodulation algorithms}

Fringe parameter estimation is limited by photon noise. Noise propagation for the phase and visibility estimators is an intrinsic characteristic of each algorithm derived from the modulation function $\Psi$. Except for the $\mathrm{DFT}_{K}$ algorithm, noise propagation for visibility and phase estimators depends on $\Phi$. By the choice of the modulation function $\Psi$, it is possible for a given phase position $\Phi$ to reduce noise in the estimated phase while increasing noise in the visibility. Such a strategy is, of course, welcome for a fringe tracker. Cophasing performance is given by the standard deviation $\sigma_{\Phi}$ of the phase estimator at $\Phi=0$. When $N V^{2} \gg 1$ :

$\sigma_{\Phi}=\frac{P_{\Phi}}{V \sqrt{N M}}$

where $N$ is the number of photoevents per modulation period and $M$ is the number of averaged modulation 
periods. $P_{\Phi}$ is a numerical coefficient depending on the modulation $\Psi$.

Visibility estimators, based on the squared amplitude of the signal, are biased. A $V^{2}$ estimator (denoted $G^{2}$, Eq. (5) in Sect. 4) is preferred to a $V$ estimator since it can be unbiased even when $V=0$. For fringe search, the figure of merit is the visibility noise out of the coherence area. In this case for any algorithm, we have (Cassaing et al. 1995):

$\left.\sigma_{G^{2}}\right|_{V=0} \simeq \frac{P_{G}}{N \sqrt{M}}$

where $P_{G}$ is a numerical coefficient depending on the modulation $\Psi$. It can be shown that the $\mathrm{DFT}_{K}$ algorithm is the one with the minimum visibility noise. Since the total number of photoevents involved is $N M$, Eq. (4) shows that it is necessary for the fringe search to increase as much as possible the number of photoevents per modulation period.

With continuous modulations, the OPD variation during the integration is equivalent to a blur, reducing the contrast of the detected interferogram. When limited by photon noise, it is thus more efficient to often read or sample the detector signal $(K \gg 1)$ to reduce the visibility loss $\eta$. For the $\mathrm{DFT}_{K}$ algorithm, $\eta=\sin (\pi / K) /(\pi / K)$, $P_{\Phi}=\sqrt{2} / \eta$ and $P_{G}=4 / \eta$. For ABCD, $P_{\Phi}=1.57$ and $P_{G}=4.44$.

Other sources of noise on $V$ and $\Phi$ are related to the turbulence perturbations on the two apertures: the high order wavefront phase distorsions (higher than OPD) are not negligible because in our experiments $r_{0}$ is always smaller than $D$, the telescope diameter. Scintillation also contributes to the noise because such intensity fluctuations cannot be distinguished from the signal resulting from the fringe temporal modulation. The correlation length of the intensity fluctuations $\sqrt{\lambda h}$ is of the order of $10 \mathrm{~cm}$ for a turbulence altitude $h \simeq 10 \mathrm{~km}$ (Fante 1975). The aperture averaging effect is therefore much lower for the I2T than for large telescopes (Roddier 1981).

\subsection{SIMONE algorithm}

The formalism of Sect. 3 can be applied to a sinusoidal modulation $\Psi=m \sin \omega t$. There is no simple closed-form expression for noise coefficients. But assuming a large $K$ value, required for best performance as previously shown, $P_{\Phi}$ and $P_{G}$ can be closely approximated by the asymptotic case $K \mapsto \infty$. For best tracking, the minimum value $P_{\Phi}=$ 1.19 is reached with a modulation amplitude $m=1.91$, i.e. $0.6 \lambda$; but then $P_{G}$ is $21 \%$ worse than with the $\mathrm{DFT}_{\infty}$ algorithm.

Other interesting values are when $J_{0}(m)=0\left(J_{0}\right.$ being the Bessel function of the first kind): the waveforms 1, $\cos \Psi$ and $\sin \Psi$ are then orthogonal (Cassaing 1997). Demodulation is therefore easily achieved using these normalized waveforms. Although not used elsewhere to our knowledge, open loop phase and visibility estimations are possible with sinusoidal modulation. We chose the first root of $J_{0}(m=2.40$, i.e. roughly $0.75 \lambda)$ and called this algorithm $\mathrm{SIMONE}_{K}$ (Sinusoidal Integrated Modulation on ONE fringe). We used $K=16$ buckets per modulation period for the sinusoid generation and the intensity demodulation, so that $\eta \simeq 1$. Noise propagation coefficients for $\mathrm{SIMONE}_{\infty}$ are $P_{\Phi}=1.27$ and $P_{G}=4.36$. $\mathrm{SIMONE}_{\infty}$ is therefore $10 \%$ better for phase tracking but $9 \%$ worse for fringe search than the $\mathrm{DFT}_{\infty}$ algorithm. The SIMONE algorithm provides good noise behavior, very similar or even better than the ABCD algorithm. An advantage of SIMONE is that high frequency sinusoidal modulation is much simpler to implement in the modulating device than triangle modulation. We used this single algorithm for fringe search and tracking.

\subsection{MAXSIM algorithm}

Since the amplitude of the phase perturbation to be corrected for can reach a few tens of fringes, whereas the rejection is limited by photon noise, phase residuals may go beyond ] $-\pi, \pi]$. This introduces phase wrapping and fringe jumps with cophasing estimators. Coherencing estimators, based on a polychromatic analysis, are then required. If the modulation amplitude is larger than $\lambda$ but smaller than $L_{\mathrm{c}}$, the envelope can be approximated by its local slope (Cassaing 1997). Reporting the first order Taylor expansion $V(\Phi+\Psi) \simeq V(\Phi)+\Psi V^{\prime}(\Phi)$ in Eq. (1) allows the measurement of $V^{\prime}(\Phi) / V(\Phi)$ in addition to the phase measurement, as described in Sect. 3, and thereby the identification of the central fringe position. Therefore, with a sinusoidal modulation of amplitude between $\lambda$ and $L_{\mathrm{c}}$, it is possible to simultaneously derive a cophasing and a coherencing estimator. If $m$ is a root of $J_{0}$, a visibility estimator can also be simply derived. We used this algorithm with $m=11.79$, i.e. $3.75 \lambda$, a third of the coherence length, and called it MAXSIM. Noise propagation coefficients for MAXSIM are similar to those of the DFT algorithm when the modulation amplitude for both is large. But in this case, the visibility is reduced because of the chromatic envelope degrading the phase measurement performance. So in Sect. 5, we will show that MAXSIM was successful in the laboratory but failed on the sky.

\section{Visibility losses of the I2T-ASSI interferometer}

\subsection{The two visibility estimators used in ASSI}

In the fringe tracker system, a pupil plane coaxial beam combination is used and the flat tint is recorded using a short exposure. This allows only one component of the object spectrum to be measured at $\mathbf{B} / \lambda$, leading to the visibility estimator $G$ defined as (Rousset et al. 1991):

$G=\frac{\left\langle\left|T_{12}(\mathbf{k}=\mathbf{0})\right|^{2}\right\rangle^{1 / 2}}{\left\langle\left|T_{\mathrm{t}}(\mathbf{k}=\mathbf{0})\right|^{2}\right\rangle^{1 / 2}}$

where \langle\rangle represents the ensemble average, $T_{12}$ is the intercorrelation of the fields of the two apertures, $T_{\mathrm{t}}$ 
the transfer function of one telescope and $\mathbf{k}$ the spatial frequency.

In the scientific instrument, the fringes are obtained by a multiaxial beam combination in a focal plane and then are recorded using a short exposure. In the data reduction process the spatial information is averaged over the fringe peak. Therefore, the measured visibility corresponds to the definition of $R_{1}$ introduced by Roddier \& Léna (1984):

$R_{1}=\frac{\iint\left\langle\left|T_{12}(\mathbf{k})\right|^{2}\right\rangle \mathrm{d}^{2} \mathbf{k}}{2 \iint\left\langle\left|T_{\mathrm{t}}(\mathbf{k})\right|^{2}\right\rangle \mathrm{d}^{2} \mathbf{k}}$.

The $R_{1}$ estimator is well adapted to a single mode interferometer, i.e. $D<r_{0}$. This does not exactly correspond to our observing conditions. A new multimode visibility estimator was recently proposed for multiaxial and short exposure modes with large apertures $\left(D \gg r_{0}\right)$ (Mourard et al. 1994). However, this estimator is not easily applicable to the I2T interferometer because the telescope diameter is only slightly larger than $r_{0}$. For the purpose of the comparison of the different visibilities we define the estimator $R_{2}=\sqrt{2 R_{1}}$ (equal to 1 when there is no aberration).

\subsection{Analysis of the visibility losses}

Various factors affect the fringe visibility in the interferometer (Cassaing et al. 1996; Robbe 1996). Atmospheric turbulence is the factor which theoretically most affects the visibility. The different contributors are:

- High order wavefront perturbations other than tiptilts and OPD. We use a numerical simulation to evaluate their effect on the estimators $G$ and $R_{2}$. Neglecting scintillation, we generate 1024 turbulent phase wavefronts for each pupil using the simulation tool of Rousset et al. (1991). Each wavefront, $j$, is a linear combination of 230 Zernike polynomials $Z_{i}(i \geq 4)$. The Zernike expansion coefficients $a_{i}{ }^{(j)}$ are Gaussian random variables obeying Kolmogorov statistics. The variances, $\sigma_{a_{i}}^{2}$, behave as $\left(D / r_{0}\right)^{5 / 3}$;

- Tip-tilt residuals. Tips and tilts are assumed to be uncorrelated between the two telescopes. In order to take into account the angle of arrival correction brought by the ASSI table, the temporal power spectrum of the tip and tilt modes (see Fig. C.1 for an outer scale $L_{0}=40 \mathrm{~m}$ ) is filtered by the transfer function of the star tracker (Robbe et al. 1997), assuming that this process is free from noise propagation. The results are added to the above computed wavefronts in order to derive $T_{12}$ and $T_{\mathrm{t}}$;

- OPD residuals. Because $R_{2}$ and $G$ are short exposure estimators, they are theoretically not affected by OPD residuals left by the fringe tracker. For $G$, the exposure is very short $(3 \mathrm{~ms})$, while much longer for $R_{2}$ (20 ms). From the temporal power spectrum of the OPD (Fig. C.1, for $L_{0}=40 \mathrm{~m}$ ) and the filtering due to the $20 \mathrm{~ms}$ exposure of the scientific instrument and the transfer function of the fringe tracker (Fig. 6), the

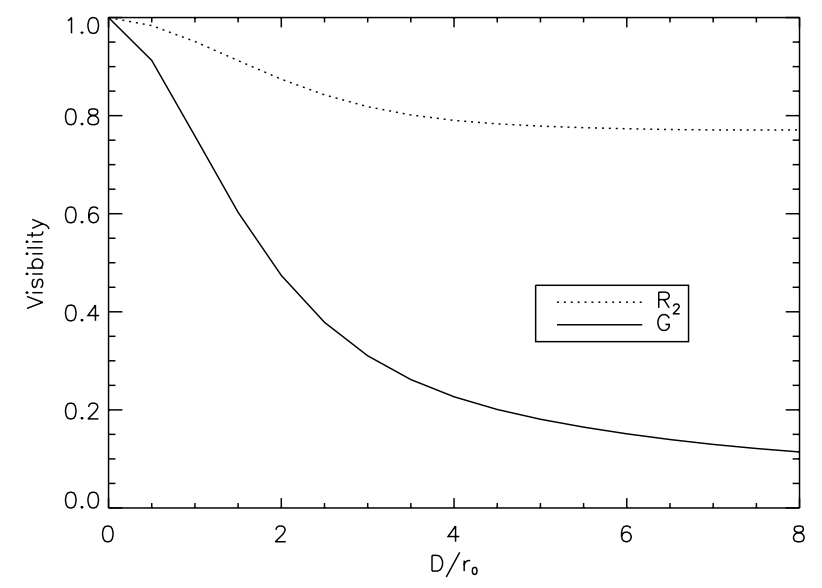

Fig. 2. Variation of the visibility estimators versus $D / r_{0}$ - tiptilt corrected by ASSI but with residuals included. No OPD residuals

high frequency OPD variance $\sigma_{\Phi \mathrm{HF}}^{2}$ can be computed. The $R_{2}$ visibility loss is estimated by $\exp \left(-\sigma_{\Phi \mathrm{HF}}^{2} / 2\right)^{2}$ (Conan \& Rousset 1995) to a value of 0.97;

- Scintillation effects (not quantified). Visibility noises are due to intensity fluctuations on the two apertures.

Estimators $G$ and $R_{2}$ are then computed for various values of $D / r_{0}$ using Eqs. (5) and (6). Figure 2 presents the results of the simulations. At large $D / r_{0}, R_{2}$ tends to $\sqrt{2 / 3}$, as theoretically predicted (Roddier \& Léna 1984). $G$ quickly decreases with an increase in $D / r_{0}$ for $D / r_{0} \leq 3$, i.e. when the first turbulence aberrations become significant in the visibility loss. For larger $D / r_{0}$, its value is low and still decreases. Because turbulence aberrations degrade the transfer function $T_{\mathrm{t}}(\mathbf{k})$ for $\mathbf{k} \neq \mathbf{0}$ but has no impact on the central value $T_{\mathrm{t}}(\mathbf{k}=\mathbf{0})$, the degradation of $T_{12}(\mathbf{k})$ is partly compensated in $R_{2}$ while it is not the case in $G$ according to Eqs. (5) and (6). Therefore, $R_{2}$ is less sensitive to the turbulence aberrations than $G$ (as shown in Fig. 2).

The second important factor is the effect of the static aberrations of the instrument. The aberrations of the optical train of the combining table, the delay-lines and the telescopes were measured with a Shack-Hartmann wavefront sensor, set up at different positions in the interferometer. From each set of measurements, the tip, tilt and defocus modes of the wavefront were filtered for visibility estimation. These modes are adjusted before each observation. For the contribution of the combining table to the static aberrations, the estimated $G$ (at $0.85 \mu \mathrm{m}$ ) is of the order of 0.65 , in good agreement with the visibility measured by the fringe sensor on the internal calibration source. For the whole interferometer, including the telescopes and the delay lines, the estimated values of $G$ and $R_{2}($ at $0.5 \mu \mathrm{m})$ are 0.44 and 0.86 respectively (see Table 1 ). As previously shown for turbulence aberrations (see comments in Fig. 2) $G$ is more degraded by static aberrations than is $R_{2}$. 
Table 1. Visibility attenuation factors $\left(r_{0}=10 \mathrm{~cm}\right.$ at $\left.0.5 \mu \mathrm{m}\right)$

\begin{tabular}{ccc}
\hline Visibility & $G(0.85 \mu \mathrm{m})$ & $R_{2}(0.5 \mu \mathrm{m})$ \\
\hline turbulence (without OPD) & 0.6 & 0.83 \\
OPD residuals & 1 & 0.97 \\
static aberrations & 0.44 & 0.86 \\
other effects & 0.91 & 0.91 \\
Total & 0.24 & 0.63 \\
\hline
\end{tabular}

The other factors reducing the fringe visibility are detailed in Appendix B. The impacts of all these factors on the visibility measurements are summarized in Table 1. For the coaxial set-up chosen in the fringe sensor, turbulence $\left(D / r_{0} \leq 2\right)$ and static aberrations strongly decrease the visibility $G$. Fringe tracking accuracy is thus decreased according to Eq. (3).

\subsection{Measured visibility level}

Analysis of the fringe tracker data shows that the experimental visibility $G$ varies between 7 and $30 \%$. Figure 2 and Table 1 show that for $r_{0}$ ranging between 10 and $16 \mathrm{~cm}$ at $0.5 \mu \mathrm{m}, G$ should be of the order of $24 \%$ and $35 \%$ respectively. Since $G$ is very sensitive to static aberrations, the discrepancy between theoretical estimations and experimental values is likely due to telescope focusing errors. Another contribution is the scintillation effect. The $R_{2}$ experimental values in the scientific instrument span between 55 and $70 \%$ (Robbe 1996). These results are consistent with the estimation of Table 1, also taking into account the contribution of telescope focusing errors.

\section{Fringe detection and tracking}

\subsection{Laboratory results}

The SIMONE demodulation algorithm (Sect. 3.2) was first tested in the laboratory. Figure 3 shows a typical interferogram in bad observing conditions $(V \simeq 9 \%)$. This interferogram is limited by photon noise: there are about $N=1400$ photoevents per $3.5 \mathrm{~ms}$ sampling time. Figure 3 also shows fringes outside the main lobe of the interferogram. This is due to the significant amount of chromatism before inserting the spectral filter in the fringe sensor $\left(L_{\mathrm{c}}=3.5 \mu \mathrm{m}\right.$ in Fig. 3$)$.

With the cophasing phase estimator, OPD correction was validated: a $90 \mathrm{~Hz}$ open-loop bandwidth at $0 \mathrm{~dB}$ was achieved with a $500 \mathrm{~Hz}$ modulation, in agreement with the loop simulation at high SNR. To reduce photon noise on the sky for visibility measurements, the modulation frequency was reduced to $280 \mathrm{~Hz}$ (Eq. (4)). In the conditions of Fig. 3, the servo-loop was automatically closed after fringe detection and was very stable at a $40 \mathrm{~Hz}$ open-loop bandwidth. No fringe jumps were observed. But in the laboratory no turbulence was simulated.

Visibility estimation was also validated. The visibility squared profile is plotted with $M=25$ in Fig. 3. The coherence area is clearly detected. The secondary peaks
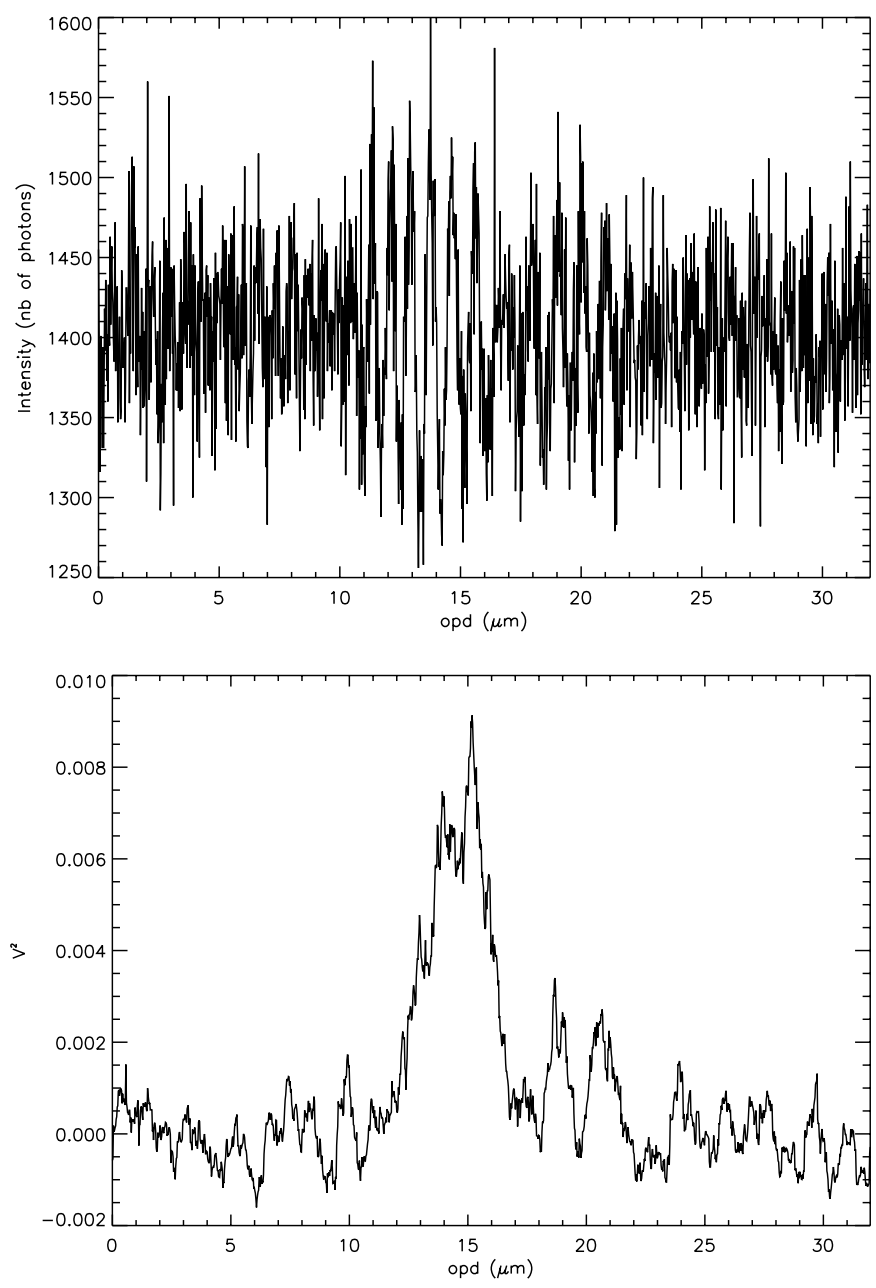

Fig. 3. Interferogram obtained from the detected intensity (top) and unbiased squared visibility (bottom) versus OPD scan, in the laboratory, with representative observing conditions $(V \simeq 9 \%, 392000$ photoevents/s)

are due to the fringes in the secondary lobe of the interferogram. Negative values of the $V^{2}$ estimator may be surprising. This is, however, required for an unbiased estimator to have a null expectation outside the coherence area. $V^{2}$ variance is about $3.510^{-7}$ out of the coherence area, in agreement with Eq. (4).

The MAXSIM algorithm was also tested. The MAXSIM phase estimation for cophasing performs as well as with SIMONE. The coherencing estimator worked successfully with high visibility $(\geq 30 \%)$. But by varying the fringe visibility, the coherencing signal turned out to be very noisy for small visibility levels since only a small portion of the envelope is scanned. It would require averaging the coherencing signal over a few seconds to achieve a sufficient SNR, but such a method was not tested.

\subsection{Conditions of observation}

Observations presented in this paper were made on the nights 7, 8, 21 and 23 October 1995 using the stars: $\alpha$ Cyg $\left(m_{\mathrm{V}}=1.25\right), \alpha \operatorname{Per}\left(m_{\mathrm{V}}=1.79\right), \beta$ Per $\left(m_{\mathrm{V}}=2.09\right)$ 


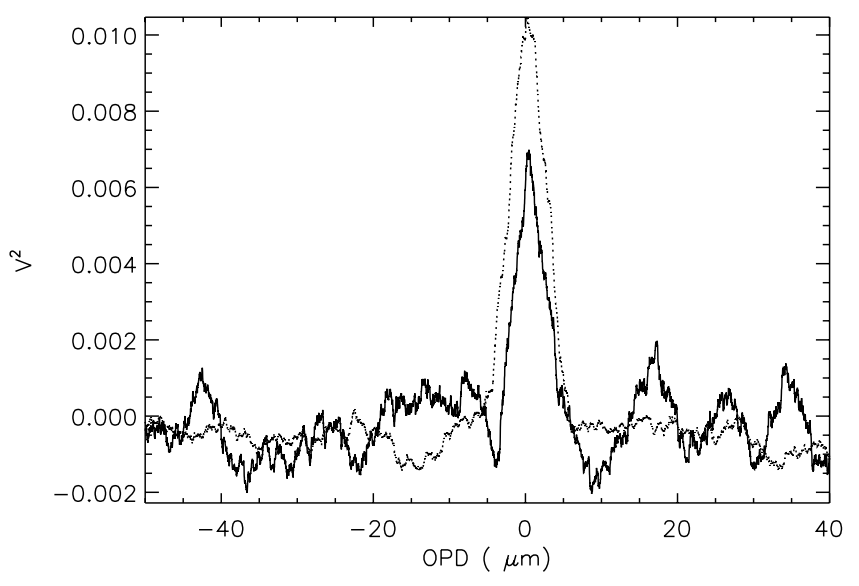

Fig. 4. Typical visibility squared profiles obtained on 23 October 1995, 19:45 UT ( $\epsilon$ Cyg, solid) and on 24 October, 00:27 UT ( $\alpha$ Per, dotted)

and $\epsilon \operatorname{Cyg}\left(m_{\mathrm{V}}=2.48\right)$. Although the I2T baseline extends up to $140 \mathrm{~m}$, all the measurements were made at a $15 \mathrm{~m}$ baseline to eliminate misalignment problems due to the displacement of the pupil conjugation optics (Robbe et al. 1994). Since the diameter of the collimated beams emerging from the telescopes $(11 \mathrm{~mm})$ and the free atmosphere path (around 7 meters) are small, the turbulence effects induced by the beam horizontal propagation from the telescopes to the central laboratory are small.

Glass plates were installed between the telescopes and the beam combining table to compensate for longitudinal chromatism. The optimal thickness of the glass plates was calculated in order to reduce the standard deviation of the optical phase difference in the red spectral bandwidth (Sorrente et al. 1994), and thus maximizing the measured fringe visibility in the fringe sensor.

Fringe visibility and OPD were recorded with a $280 \mathrm{~Hz}$ sampling frequency and the length of the data acquisition buffer typically corresponds to $3 \mathrm{mn}$.

\subsection{Fringe detection}

The first step is to detect the coherence area. Two stellar visibility profiles, similar to that of Fig. 3, are shown in Fig. $4\left(L_{\mathrm{c}}=6.5 \mu \mathrm{m}\right)$. The two stars are unresolved for the baseline used. The maximum visibility for $\alpha$ Per, observed later in the night $(00: 27 \mathrm{UT})$, is higher than for $\epsilon \mathrm{Cyg}$ (19:45 UT). The turbulence strength decreased during the night since the coherence time $t_{0}$ (see Eq. (C.3)) was of the order of $5 \mathrm{~ms}$ in the beginning of the night and reached $10 \mathrm{~ms}$ in the middle of the night. Since the goal of the fringe tracker is not to provide precise visibility measurements, we did not investigate calibration of the measured visibilities. Performance of the fringe detection is primarily driven by off-fringe visibility fluctuations. One can notice this level in Fig. 4: for $\epsilon \mathrm{Cyg}$, the secondary peaks are due to noise and not to chromatism (like in Fig. 3) because of the use of a spectral filter. The standard deviation of the measured visibility
$G^{2}$ is $\sigma_{G^{2}} \simeq 7.110^{-4}$, whereas the theoretical value is from Eq. (4) $\sigma_{G^{2} \text { th }} \simeq 1.610^{-4}(N \simeq 360$ photoevents per modulation period and $M=280$ averaged periods). For $\alpha$ Per, $\sigma_{G^{2}} \simeq 3.410^{-4}$ whereas $\sigma_{G^{2} \text { th }}=0.710^{-4}(N \simeq 450$ and $M=1440)$. This shows that visibility noise is much higher than expected; we think this results from scintillation effects (Sect. 3.1). Since this effect has been understood lately, no dedicated measurement was made. But it may a posteriori explain why, in similar photometric conditions, the off-fringe mean visibility level reached $14 \%$ on 28 September 1995 whereas the maximum off-fringe visibility during the other nights in October was a few percent. Scintillation could have been reduced without increasing photon noise (Eq. (4)) by multiplying the modulation frequency by an integer factor $p$ and extracting the phase after averaging, in intensity, $p$ successive periods.

The automatic detection algorithm performed well. In routine operation, good knowledge of the instrument allowed considerable reduction of search time and thus false alerts. The smallest detected visibility during the observations was a few percent, but no dedicated measurement for ultimate sensitivity determination was done.

\subsection{Tracking performance}

In the cophasing mode, MAXSIM and SIMONE have similar behaviour on the sky. Unfortunately, it turned out during the few tests we made that the coherencing mode of MAXSIM did not achieve the required performance because the visibility in the experiment was too low (Sect. 4). This algorithm was thus not characterized. All the data of this paper were collected with the SIMONE algorithm.

In closed-loop, the $3 \mathrm{mn}$ records are characterized by sequences where the fringe tracker loses and recovers the coherence area after a few fringe jumps. Because in the laboratory the loop was perfectly stable with a similar light level without turbulence, the fringe jumps are due to the large amplitude of the turbulent OPD fluctuations $\left(\simeq 10 \mu \mathrm{m}\right.$ rms from Eq. (C.1) for $B=15 \mathrm{~m}, r_{0}=10 \mathrm{~cm}$ and $\left.L_{0}=40 \mathrm{~m}\right)$. Locked sequences typically last $20 \mathrm{~s}$ from a fringe recovery to the next loss. However, we stress that after fringe detection, the fringe tracker was able to operate for more than one hour on the same star, in spite of the partial losses of the coherence area which occur.

In order to determine the performance of the servo and to estimate the turbulence parameters from the recorded data, we only extract data sets where the fringe tracker is locked on the fringes and where the visibility remains quasi stable $( \pm 1 \% \mathrm{rms})$, i.e. without fringe jumps. This is a very restrictive selection. Such data sets last typically $10 \mathrm{~s}$, but some reach $20 \mathrm{~s}$. In such conditions, only a few sets of measurements give information at very low temporal frequency. Figure 5 illustrates the fringe tracker operation for a visibility of $19 \%$. The recorded signals $y(t)$, the PZT actuator position, and $e^{\prime}(t)$, the error signal, are plotted. They approximate the turbulent OPD and the residual OPD error respectively (Appendix A). 

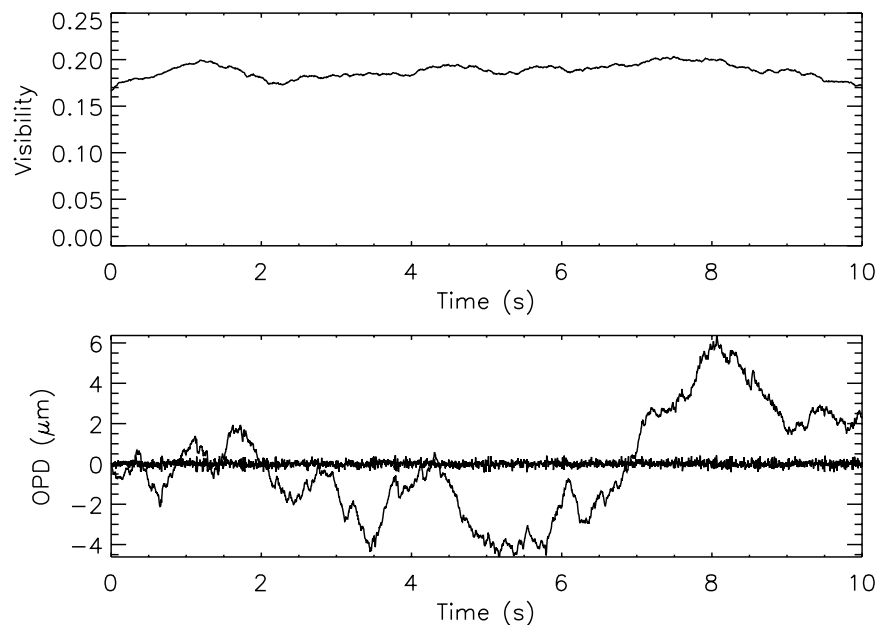

Fig. 5. Visibility versus time when the fringe tracker is active (top), $V \simeq 19 \%$. Simultaneously measured turbulent OPD, $y(t)$, and residual OPD, $e^{\prime}(t)$, versus time (bottom). Rms accuracy of the servo: $0.081 \mu \mathrm{m}$ for a turbulent OPD of $2.6 \mu \mathrm{m}$ rms. Curves obtained on 21 October 1995, 01:01 UT, star: $\alpha$ Per

A significant amount of OPD fluctuation is removed, while the visibility remains quasi constant, showing that the fringe tracker is well locked. The small visibility fluctuations can be attributed to photon noise, seeing variations (Baldwin et al. 1994) and the effect of scintillation. The reduction of the OPD fluctuations is of the order of $30 \mathrm{in}$ terms of standard deviation in Fig. 5. All the low frequencies are well filtered out.

Figure 6a shows a power spectrum of the turbulent OPD, as approximated by the PZT actuator position $y(t)$, measured over $25 \mathrm{~s}$. Figure $6 \mathrm{~b}$ shows the corresponding power spectrum of the error signal $e^{\prime}(t)$. This spectrum is flat at high frequency because of photon noise. The noise level is given by the horizontal line. The achieved accuracy is $0.1 \mu \mathrm{m}$ for a turbulence characterized by $t_{0}=12.4 \mathrm{~ms}$ at $0.85 \mu \mathrm{m}$ and 150500 photoevents/s. The open loop transfer function $|G(f)|^{2}$, plotted in Fig. 6c, is calculated as the power spectrum ratio of $y(t)$ by $e^{\prime}(t)$ (Appendix A). The experimental transfer function is very close to the $f^{-2}$ theoretical law of an integral corrector as a feedback controller. In Fig. 6c the open-loop bandwidth at $0 \mathrm{~dB} f_{\mathrm{ol}}$ is $20 \mathrm{~Hz}$.

The performance of the fringe tracker is measured by the standard deviation $\sigma_{e}$ of the true OPD residual error $e(t) . \sigma_{e}^{2}$ is derived from the variances $\sigma_{e^{\prime}}^{2}$ of the error signal measured by the fringe sensor and $\sigma_{n}^{2}$ of the noise estimated at high frequency on the error signal spectrum (Appendix A). Figure 7 presents the measurements of the rms residual error $\sigma_{e}$ and the rms noise $\sigma_{n}$ (photon noise) versus the measured visibility. As seeing conditions may rapidly change during observation, we plot $\sigma_{e}$ and $\sigma_{n}$ measured on fringe-locked sequences with various visibility levels for four records of $3 \mathrm{mn}$. The conditions of the measurements are summarized in Table 2 . For visibilities spanning between 7 and 24\%, an average OPD accuracy
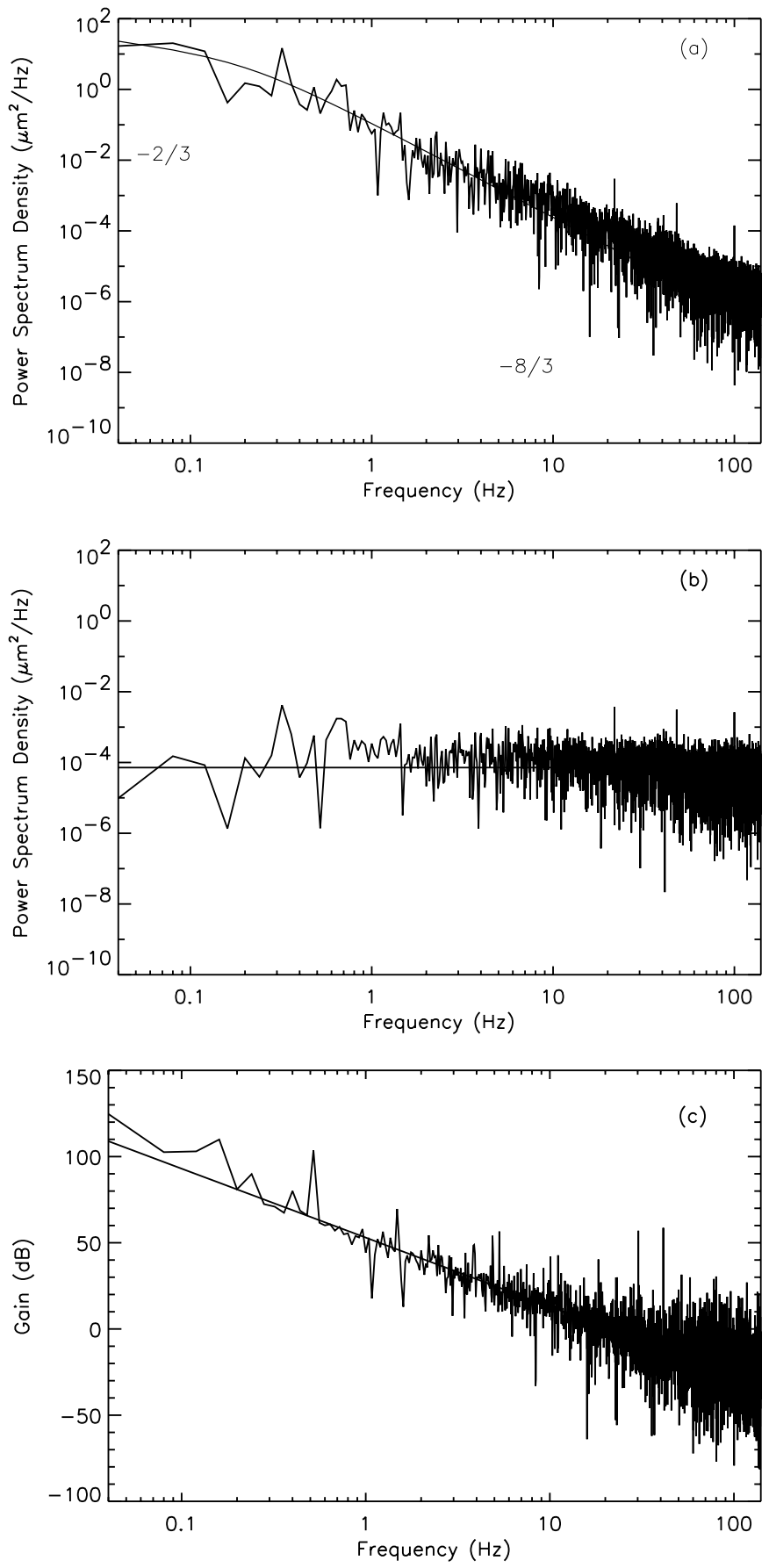

Fig. 6. Power spectrum densities of a) the PZT actuator position $y(t), \mathbf{b})$ the error signal $\left.e^{\prime}(t), \mathbf{c}\right)$ open loop transfer function $|G(f)|^{2}$ of the fringe tracker. Measured on 7 October 1995, 19:05 UT, star: $\alpha$ Cyg. Curves fitted by: a) $f^{-2 / 3}$ and $f^{-8 / 3}$ laws, b) level of white noise, c) $f^{-2}$ law

$\left(\sigma_{e}\right)$ of $85 \mathrm{~nm}(\lambda / 10$ at $0.85 \mu \mathrm{m})$ is achieved. From Fig. 7 and Table 2 several comments can be made:

- As given in Eq. (3), $\sigma_{n}$ follows the $V^{-1}$ law. The dependence in $N^{-1 / 2}$ is also roughly verified;

$-\sigma_{e}$ does not depend on the visibility $V$. Note that $\sigma_{e}^{2}$ is the sum of two contributions: the turbulence uncompensated residual OPD and the part of the photon noise propagated through the servo-loop 


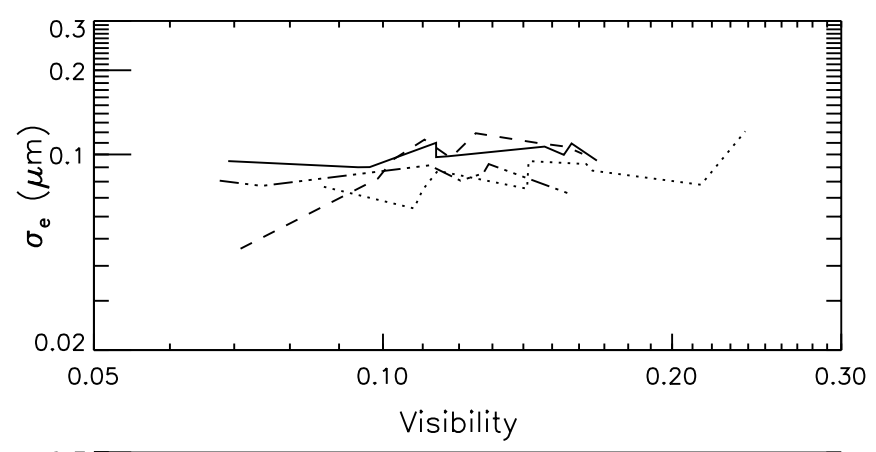

Table 2. Conditions of measurements of Fig. 7. The flux corresponds to the number of photoevents per second. $f_{\mathrm{ol}}$ is the open-loop bandwidth at $0 \mathrm{~dB}$. Coherence time $t_{0}$ given at $0.85 \mu \mathrm{m}$ (Eq. (C.2))

\begin{tabular}{ccccc}
\hline Curve & $a$ & $b$ & $c$ & $d$ \\
\hline Date $(1995)$ & $10 / 7$ & $10 / 23$ & $10 / 21$ & $10 / 23$ \\
Star & $\alpha \mathrm{Cyg}$ & $\alpha \mathrm{Cyg}$ & $\alpha \mathrm{Per}$ & $\beta \mathrm{Per}$ \\
$N$ (photoevents/s) & 150500 & 127200 & 100700 & 58600 \\
$f_{\text {ol }}(\mathrm{Hz})$ & 20 & 40 & 40 & 50 \\
$t_{0}(\mathrm{~ms})$ & 12.3 & 13.9 & 4.5 & 8.1 \\
\hline
\end{tabular}

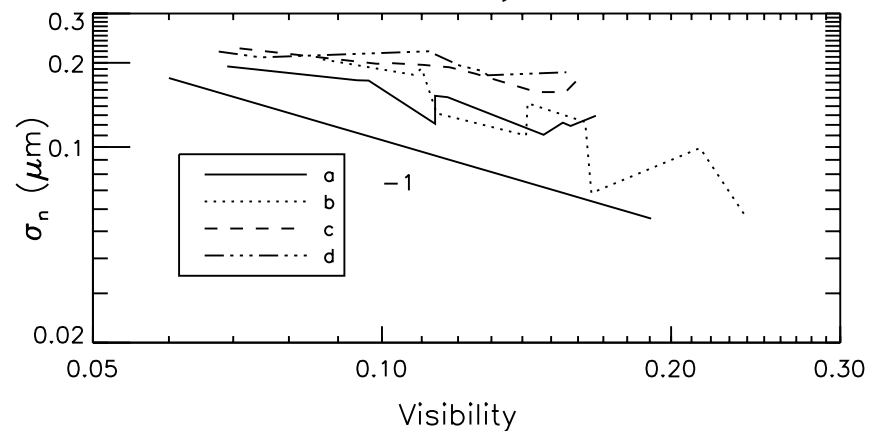

in the following conditions: $m_{H}=15$ (80000 photoevents $/ \mathrm{s}$ ), $15 \%$ fringe visibility, $r_{0}=14 \mathrm{~cm}$ at $0.5 \mu \mathrm{m}, v=$ $10 \mathrm{~m} \mathrm{~s}^{-1}, L_{0}=40 \mathrm{~m}$ and $50 \mathrm{~Hz}$ bandwidth. For a scientific observation made at $2.2 \mu \mathrm{m}$, the tracking performance is then of the order of $\lambda / 22$. We stress that the limiting magnitude depends on the specified OPD accuracy and on the seeing conditions.

Fig. 7. Accuracy, $\sigma_{e}$ (top), and rms noise $\sigma_{n}$ (bottom), versus fringe visibility in $\log -\log$ scales for four runs detailed in Table 2. A line with slope of -1 is shown

(Appendix A). Only the second contribution depends on the visibility (as $\sigma_{n}$ ). For the considered light levels, the accuracy $\sigma_{e}$ is dominated by the turbulence residuals. This is due to the servo-loop bandwidth being too low, i.e. the loop-gain which was crudely optimized by the observer was too low (Sect. 2.2). Indeed in Fig. 7 and Table 2, the comparison of Curves $b$ and $c$ on one hand and of Curves $a$ and $d$ on the other hand shows the performance dependence on the coherence time and the bandwidth, respectively. Moreover, when the number of photoevents decreases by a factor of 2 to 3 , no clear evidence of a loss of accuracy is shown.

No tests of limiting magnitude have been performed. Nevertheless, fringes have been stabilized on a $2.48 \mathrm{mag}$ nitude star $(\epsilon \mathrm{Cyg})$ with an accuracy of the order of $\lambda / 10$ at $0.85 \mu \mathrm{m}$ for $t_{0}=8 \mathrm{~ms}$, an overall optical throughput estimated to be of the order of $0.8 \%$ and a $80 \mathrm{~nm}$ spectral bandwidth. Using Eqs. (A.1) and (A.3) and the measured observing conditions, we checked by simulation (as in Sect. 4.2) that the experimental performance $\left(\sigma_{e}\right)$ was close to the theoretical one. If all the coatings of the optical surfaces were optimized, the throughput would have reached $10 \%$ and the $\lambda / 10$ accuracy would have been achieved on a 5 magnitude star in the I2T-ASSI configuration for the same turbulence conditions.

Using the same simulation, it is possible to evaluate the performance of the fringe tracker presented here for an interferometer working with $8 \mathrm{~m}$ apertures partially corrected by adaptive optics (Rousset et al. 1991) and a $40 \mathrm{~m}$ baseline. The fringe tracking technique working in $H$ band would lead to a $100 \mathrm{~nm}$ OPD accuracy

\section{Seeing characterization}

\subsection{Fringe tracker measurements}

The fringe tracker bandwidth is sufficiently large to check the agreement of the data with the Kolmogorov model (see Appendix C) at frequencies lower than $20 \mathrm{~Hz}$, as illustrated in Fig. 6a. A fit with $-2 / 3$ and $-8 / 3$ power laws is superimposed.

We determined the high frequency slope of 30 power spectra of $y(t)$. They have been processed as follows: an autoregressive filter (Makhoul 1975) is first estimated from the temporal data. Then, a polynomial fit of the filter is made. The slope of the high frequency part of the spectrum is determined at the inflection point of the polynomial fit. The main advantage of this method is that the slope at the inflection point is less corrupted than at other points of the spectrum by the low frequency behavior and by the noise appearing at very high frequency.

The average value is $-7.84 / 3$ with a dispersion of \pm 0.17 . We conclude that the $-8 / 3$ regime predicted by the Kolmogorov model (Appendix C) is in good agreement with our measurements. From all our data, no evidence of a $-17 / 3$ regime was observed for $f>0.3 v / D(\simeq 10 \mathrm{~Hz}$ for $v=10 \mathrm{~m} \mathrm{~s}^{-1}$ ) although the bandwidth of the servo can be as large as $50 \mathrm{~Hz}$ in some records. We think this results from the aliasing of the high order phase distorsions in the OPD measurement by temporal modulation. Indeed, the high order phase distorsions have temporal frequency spectra spanning higher frequencies than the OPD one (Conan et al. 1995).

According to Eq. (C.2), $t_{0}$ can be calculated from the fit of the high frequency part of the fringe motion spectra with the autoregressive filter technique. Mean values of $t_{0}$ corresponding to four nights of observations are quoted in Table 3 . The coherence time was typically equal to $9 \mathrm{~ms}$ in the $0.81-0.89 \mu \mathrm{m}$ spectral range of the fringe sensor. 
Table 3. Mean values and rms dispersions of the coherence time $t_{0}$ (Eq. (C.2)) at $0.85 \mu \mathrm{m}$ obtained from the fringe tracker (top). Mean values and rms dispersions of $r_{0}$ at $0.5 \mu \mathrm{m}$ and $v$ obtained from the star tracker and $t_{0}$ calculated at $0.85 \mu \mathrm{m}$ from Eq. (C.3) (bottom). $M$ is the number of data sets used

\begin{tabular}{ccccc}
\hline Date & $10 / 7 / 95$ & $10 / 8 / 95$ & $10 / 21 / 95$ & $10 / 23 / 1995$ \\
\hline$t_{0}(\mathrm{~ms})$ & $11.9 \pm 1.9$ & $8.6 \pm 2.2$ & $8.6 \pm 3.3$ & $9.2 \pm 1.6$ \\
$M$ & 9 & 6 & 30 & 10 \\
\hline$r_{0}(\mathrm{~cm})$ & $18.4 \pm 3.8$ & $8.9 \pm 1.6$ & $16.4 \pm 3.9$ & $12.3 \pm 2.3$ \\
$v\left(\mathrm{~m} \mathrm{~s}^{-1}\right)$ & $12 \pm 4.2$ & $6.1 \pm 1.3$ & $9.7 \pm 1.6$ & $11.4 \pm 5.5$ \\
$t_{0}(\mathrm{~ms})$ & $9.8 \pm 3.0$ & $8.9 \pm 1.9$ & $10.5 \pm 3.6$ & $7.9 \pm 3.3$ \\
$M$ & 6 & 7 & 8 & 4 \\
\hline
\end{tabular}

\subsection{Star tracker measurements}

According to Appendix C, $r_{0}, v$ and $L_{0}$ can be estimated from the variance and the power spectrum of the angle of arrival. $r_{0}$ and $v$ estimations have already been published in the first paper (Robbe et al. 1997). Autoregressive filters are used to fit angle of arrival spectra in order to determine the seeing parameters. Figure 8 shows a power spectrum obtained from a $3 \mathrm{mn}$ long data record of the $x$ axis of the south tip-tilt mirror. The telescope drift is removed from the data after checking that no mirror actuator desaturation occurs during the record. The fitting of the spectrum of Fig. 8 with an autoregressive filter shows $f^{-2.4 / 3}$ and $f^{-10 / 3}$ laws close to the theory (Fig. C.1). For all the data sets of the four nights the measured average slopes are $-2.2 / 3 \pm 0.2$ at low frequency and $-10.2 / 3 \pm 0.2$ at high frequency. This confirms the evidence of the Kolmogorov behavior for frequency higher than $0.2 \mathrm{~Hz}$. However, the most interesting point is at very low frequency: the clear deviation from the Kolmogorov model, peculiar to a finite outer scale. This saturation effect was observed on a large number of angle of arrival power spectra during the observations of October 1995. We checked that the flattening effect was not due to the removal of the telescope drift, simulating a temporal sequence of angle of arrival obeying the Kolmogorov model, and removing the drift due to the turbulence itself.

A $L_{0}$-independent estimation of $r_{0}$ requires an extrapolation of the $-2 / 3$ Kolmogorov behavior at very low frequency in order to compensate for the effects of the saturation due to $L_{0}$ and in addition for the finite duration of the record. The second parameter deduced from the angle of arrival is the average wind speed, $v$, estimated from the knee frequency $f_{2}$ (Appendix C). As shown in Fig. 8, the $-2 / 3$ and the $-11 / 3$ regimes can be clearly distinguished. From $r_{0}$ and $v$ we derive $t_{0}$ through Eq. (C.3). The estimated values of these parameters are gathered in Table 3. Estimations of $t_{0}$ deduced from the fringe tracker data and the angle of arrival data are in agreement. Note that the data recordings on the star tracker and the fringe tracker were generally not simultaneous, but not too much delayed.

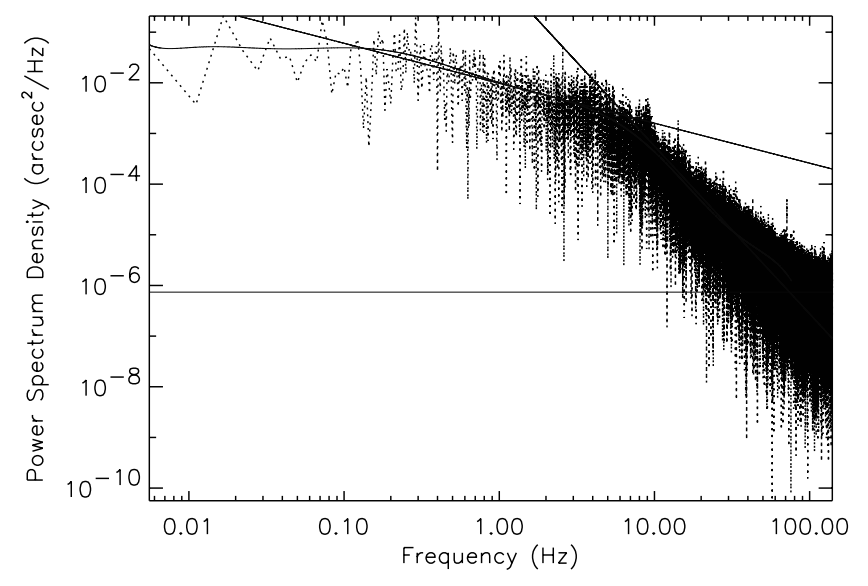

Fig. 8. Power spectrum of the angle of arrival measured on the south $x$ axis of the star tracker and fitted by an autoregressive filter. Measured on 8 october 1995, 19:19 UT, star: $\alpha$ Cyg. Horizontal line: level of white noise

\subsection{Outer scale estimation}

The analysis of the angle of arrival spectra obtained in October 1995 leads to a value of the first knee frequency, $f_{0}$, varying between 0.1 and $0.3 \mathrm{~Hz} . L_{0}$ is derived from $f_{0}$ using $v$ estimated on the same spectrum from $f_{2} . L_{0}$ typically ranges from 20 to $50 \mathrm{~m}$ during the observations. The average value is $40 \mathrm{~m}$. Such a method presents systematic errors in the estimation of the outer scale since the determination of the first knee frequency, $f_{0}$, depends on the obtained fit of the $-2 / 3$ regime.

Another way to estimate the outer scale from the angle of arrival data is to compare the experimental variance to the theoretical von-Karman variance (Eq. (C.4)). The outer scale was calculated using the instantaneous value of $r_{0}$ derived in Sect. 6.2. Values of $L_{0}$ from 1 to $120 \mathrm{~m}$ (mean value of $25 \mathrm{~m}$ ) are found. The dispersion of the measurements is more important here: obviously $L_{0}$ values are very sensitive to $r_{0}$ estimation. An error of $5 \%$ in $r_{0}$ leads to an error of $50 \%$ in $L_{0}$. Small values of $L_{0}$ are not consistent with the observed shape of the power spectra, since an outer scale of a few meters would lead to $f_{0}$ of the order of $1 \mathrm{~Hz}$. Clearly, this was never observed.

$L_{0}$ may also be estimated by comparing the theoretical von-Karman variance (Eq. (C.1)) to the experimental variance of the fringe motion, also taking into account the effect of the finite duration of the measurements. $r_{0}$ is deduced from $t_{0}$ obtained from the OPD spectrum and $v$ corresponding to the closest record of angle of arrival. From the OPD data, $L_{0}$ typically ranges between $30 \mathrm{~m}$ and $120 \mathrm{~m}$, with an average value of $50 \mathrm{~m}$. These results are consistent with the estimations derived from the angle of arrival data. Furthermore, as for the case of the angle of arrival spectra, the observed knee frequency of the OPD spectra is always close to $0.2 \mathrm{~Hz}$.

Finally, the estimates of the outer scale typically range between 20 and $120 \mathrm{~m}$. Values smaller than $10 \mathrm{~m}$ are not compatible with our observations. 


\section{Conclusion}

We have described the fringe tracker system of the ASSI table developed to compensate in real-time the I2T interferometer for the turbulent OPD. The PZT delay line is controlled by a fringe sensor based on a temporal modulation and equipped with a photon-counting APD. The free parameters of the sensor, i.e. the modulation function, the number of buckets and the demodulation algorithm have been optimized for best performance. Unlike similar systems based on triangle modulation and the DFT algorithm, a sinusoidal modulation is used. Visibility estimation or closed-loop phase measurements can be optimized by the choice of the modulation amplitude. This new algorithm, so-called SIMONE, has been tested and validated on the sky for fringe detection and cophasing. It is based on a sinusoidal OPD modulation of amplitude $0.75 \lambda$ and has performance similar to that of the ABCD algorithm. A large number of buckets (16) ensures minimum visibility loss without any extra cost. The modulation frequency is $280 \mathrm{~Hz}$. The bandwidth is adjusted by a numerical gain according to the observing conditions. In the reported experiments the bandwidth ranges between 20 and $50 \mathrm{~Hz}$.

The fringe tracker (SIMONE algorithm) achieves a typical OPD accuracy of $\lambda / 10$ at $0.85 \mu \mathrm{m}$ for a visibility ranging between 7 and $24 \%$, a coherence time $t_{0}$ around $9 \mathrm{~ms}$ and a 2 magnitude star. With optimized optical throughput, this performance would have been achieved on a 5 magnitude star. High temporal bandwidth (around $50 \mathrm{~Hz}$ in open-loop) is required to obtain good performance for bright stars. For low bandwidth or small $r_{0}$, OPD residuals are such that the cophasing algorithm suffers from fringe jumps. Fringe-locked sequences typically last $20 \mathrm{~s}$. However, the fringe tracker operation can last more than one hour without fatal loss of the coherence area. It was not possible to successfully implement the coherencing algorithm because the visibility on the sky was too low. Sometimes, scintillation was found to severely limit the system. The modulation frequency should have been higher, taking advantage of the sinusoidal modulation.

Visibility losses are estimated in the ASSI-I2T interferometer. They are mainly due to the static aberrations of the optical train and the wavefront fluctuations due to the turbulence. For the fringe sensor, the visibility is lower than $24 \%$ for $r_{0}=10 \mathrm{~cm}$ at $0.5 \mu \mathrm{m}$. This estimation is in good agreement with the measured visibilities. For the scientific instrument, the visibility is lower than $70 \%$.

Turbulence parameters are characterized for the evaluation of the observing conditions. The temporal power spectra of the fringe motion are well modeled by the Kolmogorov statistics at high frequency since an average slope of $-7.8 / 3$ has been measured for the $-8 / 3$ theoretical prediction. This expected behavior allows us to determine the coherence time, $t_{0}$. An average coherence time of the order of $9 \mathrm{~ms}$ at $0.85 \mu \mathrm{m}$ was estimated during the observations of October 1995. The agreement between the estimations of $t_{0}$ derived from the data of the star and fringe trackers underlines the reliability of the Kolmogorov model at very different scales in the inertial range: $0.26 \mathrm{~m}$ for the star trackers and $15 \mathrm{~m}$ for the fringe tracker. Our observations corresponds to average seeing conditions with $r_{0}$ ranging between 8 and $18 \mathrm{~cm}$ at $0.5 \mu \mathrm{m}$.

The star tracker data show that the angle of arrival spectra depart at very low frequency from the theoretical prediction based on the pure Kolmogorov model. Analysis of these data leads to an average outer scale of the order of $40 \mathrm{~m}$ with a range of variation between 20 and $120 \mathrm{~m}$. This estimation was confirmed by the analysis of the fringe tracker data.

Lessons learned from ASSI experiment recently have been used in the design of a new fringe tracker for the VLTI (Cassaing 2000). Since the fringe tracker generally differs from the scientific instrument, algorithms optimized for fringe tracking should be used instead of the triangle modulation of amplitude $\lambda$ optimized for visibility measurements. Moreover, spatial modulation avoids cross-talk present in temporal modulation between OPD measurement, turbulent intensity fluctuation induced by scintillation, high order wavefront distorsions and high temporal frequencies of the residual OPD. Finally, coherencing should be performed by dispersion, as in most other interferometers (Armstrong et al. 1998; Colavita et al. 1999).

Acknowledgements. The authors wish to thank the anonymous referee for his or her helpful and numerous comments, C. Dessenne for her fruitful advice in the data processing, G. Merlin for his support during the observations, C. Coudrain and L. Ménager for their participation in the fringe tracker development. This study was funded by the Direction des Recherches, Études et Techniques of the French Defense Ministry.

\section{Appendix A: Measured signals in the servo-loop}

The block diagram of the fringe tracker is illustrated in Fig. A.1. The residual OPD error, $e(t)$, seen by the scientific instrument and the fringe sensor, is the difference between the incoming OPD due to turbulence $a(t)$ and the PZT actuator position $y(t)$. $e(t)$ represents the accuracy of the servo-loop. The error signal $e^{\prime}(t)$ as measured by the fringe sensor is corrupted by white noise $n(t)$.

Let's call $G(f)$ the transfer function of the fringe tracker (including sensor, computer and delay line), where $f$ is the temporal frequency. According to Fig. A.1, the following relations hold, denoting with $\widehat{\cdot}$ the spectrum of the temporal variables:

$$
\begin{aligned}
\widehat{e^{\prime}}(f) & =\frac{\widehat{a}(f)}{1+G(f)}+\frac{\widehat{n}(f)}{1+G(f)} \\
\widehat{e}(f) & =\frac{\widehat{a}(f)}{1+G(f)}-\widehat{n}(f) \frac{G(f)}{1+G(f)} \\
\widehat{y}(f) & =[\widehat{a}(f)+\widehat{n}(f)] \frac{G(f)}{1+G(f)}
\end{aligned}
$$




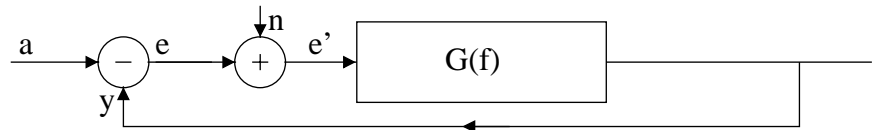

Fig. A.1. Block diagram of the fringe tracker servo

The noise $n$ is uncorrelated from the residual error $e$, $\sigma_{e^{\prime}}^{2}=\sigma_{e}^{2}+\sigma_{n}^{2}$ (Dessenne et al. 1998), thus it is possible to derive the actual performance of the system $\sigma_{e}^{2}$ since $\sigma_{n}^{2}$ can be estimated from the power spectrum of $e^{\prime}$ with an autoregressive filter. Equation (A.1) shows that $\widehat{e^{\prime}}(f)$ is composed of two terms related to $a$ and $n$. Since at high frequency $(G(f) \ll 1) a$ has a fast decreasing power spectrum (see Appendix C), then $\widehat{e^{\prime}}(f)$ tends to the white spectrum of the noise. Hence, the noise level can be estimated.

Since $G \simeq 1+G$ for $f \leq f_{\text {cl }}, f_{\text {cl }}$ the closed-loop bandwidth, $\widehat{y}(f)$ is representative of the turbulence fluctuations assuming that $\widehat{n}(f) \ll \widehat{a}(f)$ in this domain (see Eq. (A.3)). Hence, the spatial and temporal characteristics of the turbulence can be directly deduced from the signal $\widehat{y}(f)$.

Finally, Eq. (A.2) shows that it is possible to adjust the gain in $G(f)$ in order to minimize the residual error, taking into account the respective levels of turbulence and noise, i.e. the observing conditions.

\section{Appendix B: Others factors reducing the fringe visibility}

The factors other than optical aberrations which reduce the fringe visibility are:

- The field rotation attenuation factor, which depends on the star declination (Koechlin 1985). Since we chose stars close to the zenith, this factor around the transit ( \pm 1 hour is not important: typically, $\simeq 0.97$;

- Differential polarization effects. They may appear when each beam does not encounter the same sequence of reflections (Traub 1988). Measurements of the Jones matrices of the two arms revealed an important chromatic differential effect. A vertical polarizer and a $80 \mathrm{~nm}$ filter centered on $0.85 \mu \mathrm{m}$, which corresponds to a $9 \mu \mathrm{m}$ coherence length, were placed before the APD. They allow the elimination of this problem, since the north and south Jones matrices are diagonal. The main drawback is that half of the light is rejected;

- Differential chromatism (Lacasse \& Traub 1988), including longitudinal dispersion and atmospheric refraction. Differential diffraction and residual longitudinal chromatism after the glass plates were simulated. Their effect on fringe visibility is negligible since the baseline (around $15 \mathrm{~m}$ ) and the zenith angle are small. The chromatism, introduced by the refractive optics of the ASSI table, is also reduced by the use of the spectral filter;

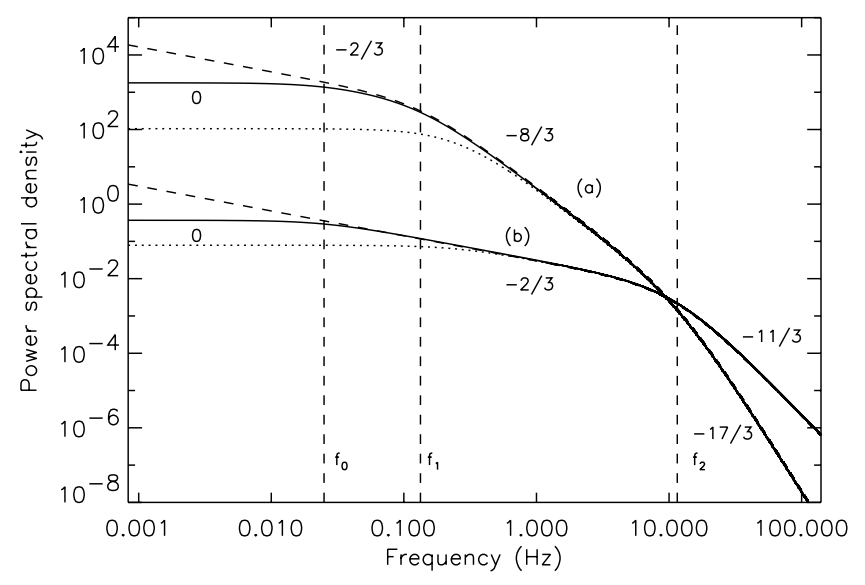

Fig. C.1. Theoretical power spectra of: a) fringe motion (in $\mu \mathrm{m}^{2} \mathrm{~Hz}^{-1}$ ) and b) angle of arrival (in $\operatorname{arcsec}^{2} \mathrm{~Hz}^{-1}$ ) for a pure Kolmogorov model (dashed line), for a von-Karman model with $L_{0}=40 \mathrm{~m}$ (dotted line) and $L_{0}=400 \mathrm{~m}$ (solid line). $r_{0}=10 \mathrm{~cm}$ at $0.5 \mu \mathrm{m}, B=15 \mathrm{~m}, D=0.26 \mathrm{~m}$ and $v=10 \mathrm{~m} \mathrm{~s}^{-1}$. The knee frequencies $f_{0}\left(\right.$ for $\left.L_{0}=400 \mathrm{~m}\right), f_{1}$, $f_{2}$ and the exponents of the power laws are given

- Differential intensity. Intensities in the north and south beams differ by a factor of 2 because of different throughputs. The attenuation factor is therefore $2 \sqrt{I_{1} I_{2}} /\left(I_{1}+I_{2}\right)=0.94$.

\section{Appendix C: Theoretical behavior of the measured parameters}

In stellar interferometry, when $B \gg D$, the variance of the turbulent phase $\Phi$ of the interferogram can be approximated by $\sigma_{\Phi}^{2} \simeq D_{\varphi}(\mathbf{B})$ with:

$D_{\varphi}(\mathbf{B})=3.69\left(\frac{r_{0}}{L_{0}}\right)^{-\frac{5}{3}}\left[1-\frac{5}{3} \frac{\left(\frac{B}{L_{0}}\right)^{\frac{5}{6}}}{2^{\frac{5}{6}} \Gamma\left(\frac{11}{6}\right)} K_{\frac{5}{6}}\left(\frac{B}{L_{0}}\right)\right]$

where $K_{\frac{5}{6}}$ is the modified Bessel function of order $5 / 6 . D_{\varphi}$ is the phase structure function computed by Valley (1979) for a modified Kolmogorov spectrum, taking into account the effect of a finite outer scale $L_{0}$ (von-Karman spectrum). Therefore, the measurement of $\sigma_{\Phi}^{2}$ by the fringe sensor can be used to estimate the two turbulent parameters $r_{0}$ and $L_{0}$, for instance by varying the baseline (Mariotti \& Di Benedetto 1984).

Considering the von-Karman model, it can be shown that the theoretical temporal power spectrum of the OPD fluctuations (Buscher et al. 1995; Conan et al. 1995) can be divided into different cases as plotted in Fig. C.1:

- At very low frequencies, $f<f_{0}=v / L_{0}$ where $v$ is the average wind speed, the spectrum flattens to a $f^{0}$ power law as the result of the finite outer scale;

- At low frequency, $f_{0}<f<f_{1}=0.2 v / B$, the conventional Kolmogorov $f^{-2 / 3}$ law holds. We stress that for outer scales of the order of a few tenth of meters, this regime is not observable in the spectrum $\left(v / L_{0} \simeq 0.2 v / B\right)$; 
- At high frequency, $f_{1}<f<f_{2}=0.3 v / D$, the spectrum shows the conventional Kolmogorov $f^{-8 / 3}$ law. In this case the phases on the two telescopes are independent, the OPD power spectrum is twice the phase power spectrum;

- At very high frequency, $f_{2}<f$, the spectrum obeys a $f^{-17 / 3}$ law.

Following Buscher (1995), the coherence time $t_{0}$ can be deduced from the vertical position of the $f^{-8 / 3}$ asymptote of the power spectrum $W_{\Phi}$, since:

$W_{\Phi}(f)=1.1210^{-2} t_{0}^{-\frac{5}{3}} f^{-\frac{8}{3}}$.

The advantage of this method is that $t_{0}$ estimation is not affected by the value of $L_{0}$. According to Taylor's hypothesis, $t_{0}$ can also be deduced from the knowledge of $r_{0}$ and the average wind speed $v$ by the relation (Roddier et al. 1982):

$t_{0}=0.314 \frac{r_{0}}{v}$.

The variance of the angle of arrival $\alpha$ on each telescope $\sigma_{\alpha}^{2}$ is directly linked to $r_{0}$ and $L_{0}$ (Fante 1975; Ziad 1993):

$$
\begin{aligned}
\sigma_{\alpha}^{2}= & 0.17 \lambda^{2} \frac{r_{0}^{-\frac{5}{3}}}{D^{\frac{1}{3}}}\left[1-1.525\left(\frac{D}{L_{0}}\right)^{\frac{1}{3}}+5.568\left(\frac{D}{L_{0}}\right)^{2}\right. \\
& -6.445\left(\frac{D}{L_{0}}\right)^{\frac{7}{3}}+0.365\left(\frac{D}{L_{0}}\right)^{3} \\
& \left.-0.367\left(\frac{D}{L_{0}}\right)^{\frac{10}{3}}+\ldots\right]
\end{aligned}
$$

As shown in Fig. C.1, the theoretical temporal power spectrum of the angle of arrival tends also to a $f^{-2 / 3}$ law at low frequency while the high frequency component is characterized by a $f^{-11 / 3}$ power law. These regimes are separated by a knee frequency $f_{2}=0.3 \mathrm{v} / \mathrm{D}$ (Conan et al. 1995). As for the case of the OPD, the power spectrum saturates at frequencies lower than $f_{0}=v / L_{0}$ and a $f^{0}$ behavior is also predicted for the von-Karman model. Even for $L_{0}=40 \mathrm{~m}$ the $f^{-2 / 3}$ regime is clearly observed in the angle of arrival spectrum but not in the OPD spectrum.

\section{References}

Armstrong, J. T., Mozurkewich, D., Rickard, L. J., et al. 1998, ApJ, 496, 550

Baldwin, J. E., Boysen, R. C., Cox, G. C., et al. 1994, in (Breckinridge 1994), 112

Beckers, J. M., Merkle, F. (ed.) 1991, ESO Proc. 39, HighResolution Imaging by Interferometry II

Bester, M., Danchi, W. C., Degiacomi, C. G., et al. 1992, Astrophys. J., 357
Breckinridge, J. B. (ed.) 1994, Proc. SPIE 2200, Amplitude and Intensity Spatial Interferometry II

Buscher, D. F., Armstrong, J. T., Hummel, C. A., et al. 1995, Appl. Opt., 34(6), 1081

Cassaing, F., Mugnier, L., Sorrente, B., et al. 1995, Technical Report 4/4625 PY, ONERA

Cassaing, F., Mugnier, L., Sorrente, B., et al. 1996, Technical Report 8/4625 PY, ONERA

Cassaing, F. 1997, Ph.D. Thesis, Université Paris XI Orsay

Cassaing, F., Fleury, B., Coudrain, C., et al. 2000, in Proc. SPIE 4006, Interferometry in optical astronomy

Colavita, M. M., Wallace, J. K., Hines, B. E., et al. 1999, Astrophys. J., 510(1)

Conan, J.-M., \& Rousset, G. 1995, in ESO Proc. 54, Topical Meeting on Adaptive Optics, 351

Conan, J.-M., Rousset, G., \& Madec, P.-Y. 1995, J. Opt. Soc. Am. A, 12(12), 1559

Damé, L., Faucherre, M., Bourdet, G., et al. 1988, in (Merkle 1988), 1079

Davis, J., Lawson, P. R., Booth, A. J., et al. 1995, Mon. Not. R. Astr. Soc., 273, L53

Dessenne, C., Madec, P. Y., \& Rousset, G. 1998, Appl. Opt., $37(21), 4623$

Fante, R. 1975, Proc. IEEE, 63(12), 1669

Koechlin, L. 1985, Ph.D. Thesis, Université de Nice

Koechlin, L., \& Rabbia, Y. 1985, A\&A, 153, 91

Kœchlin, L., Lawson, P. R., Mourard, et al. 1996, Appl. Opt., $35(16), 3002$

Labeyrie, A. 1975, ApJ, 196, L71

Lacasse, M. G., \& Traub, W. A. 1988, in (Merkle 1988), 959

Makhoul, J. 1975, in Proc. IEEE, vol. 63, 561

Mariotti, J., \& Di Benedetto, G. 1984, in Very Large Telescopes, their Instrumentation and Programs, vol. 79, 257, IAU, ed. M.-H. Ulrich, \& K. Kjär

Merkle, F. (ed.) 1988, ESO Proc. 29, High-resolution imaging by interferometry

Mourard, D., Tallon-Bosc, I., Rigal, F., et al. 1994, A\&A, 288, 675

Robbe, S. 1996, Ph.D. Thesis, Université de Nice-Sophia Antipolis

Robbe, S., Sorrente, B., Cassaing, F., et al. 1997, A\&A, 125, 1

Robbe, S., Sorrente, B., Cassaing, F., et al. 1994, in (Breckinridge 1994), 222

Roddier 1981, Progress in Optics, ed. E. Wolf, XIX, 281

Roddier, F., Gill, J. M., \& Lund, G. 1982, J. Optics (Paris), 13(5), 263

Roddier, F., \& Léna, P. 1984, J. Optics (Paris), 15(4), 171

Rousset, G., Madec, P. Y., \& Rabaud, D. 1991, in (Beckers 1988), 1095

Shao, M., Colavita, M. M., Hines, B. E., et al. 1988, A\&A, 193, 357

Sorrente, B., Cassaing, F., Beal, D., et al. 1991, in (Beckers 1988), 1133

Sorrente, B., Cassaing, F., \& Rousset, G. 1994, Technical Report 3/4625 PY, ONERA

Traub, W. A. 1988, in (Merkle 1988), 1029

Valley, G. C. 1979, Appl. Opt., 18(7), 984

Ziad, A. 1993, Ph.D. Thesis, Université Nice-Sophia Antipolis 